\title{
Compuestos volátiles libres y enlazados glicosídicamente en la pulpa de la uva Caimarona (Pourouma cecropiifolia Mart.)
}

\author{
Juliana Constanza BARRIOS GUIO ${ }^{1}$, Diana Cristina SINUCO LEON ${ }^{2}$, Alicia Lucia MORALES PEREZ ${ }^{3}$
}

\begin{abstract}
RESUMEN
Los componentes volátiles presentes en la pulpa de la uva Caimarona se estudiaron mediante GC-MS. Éstos se extrajeron por tres técnicas: evaporación del aroma asistida con solvente (Solvent Assisted Flavour Evaporation SAFE), extracción continua líquido-líquido (LL) y destilación por arrastre con vapor-extracción simultanea con solvente orgánico (DES). En general los componentes volátiles predominantes en la pulpa fueron alcoholes alifáticos y terpénicos. Las notas olfativas del extracto SAFE fueron descritas como floral tenue y verde herbal similares a las exhibidas por la pulpa fresca. Este extracto presentó como componentes mayoritarios linalol 1,2-propanodiol y salicilato de metilo. En contraste, el extracto LL presentó notas que recuerdan la uva pasa y el vino moscatel y sus componentes mayoritarios fueron el ácido acético, el salicilato de metilo y el 2,6-dimetil-2(Z),7-octadien-1,6-diol. El extracto DES fue descrito con notas fresca, floral, cereal y amargo y está constituido por un reducido número de componentes mostrando el efecto negativo de la temperatura en la extracción; sus componentes mayoritarios fueron 1,2-propanodiol, linalol y salicilato de metilo. Adicionalmente, los componentes volátiles mayoritarios liberados por hidrólisis enzimática (Rohapect D5L) de los glicósidos de la pulpa fueron ácido acético, ácido benzoico y vainillina. Cabe destacar que aunque el linalol no se encontró entre las agliconas volátiles, se detectaron los dioles biogenéticamente relacionados: 3,7-dimetil-1,5-octadien-3,7-diol y los isómeros $E$ y $Z$ del 2,6-dimetil-2,7-octadien-1,6-diol.
\end{abstract}

PALABRAS-CLAVE: Pourouma cecropiifolia; Amazon grape, Uva Caimarona; Cecropiaceae; Compuestos volátiles.

\section{Compostos voláteis livres e enlaçados glicosídicamente na polpa da uva Caimarona (Pourouma cecropiifolia Mart.)}

\begin{abstract}
RESUMO
Os componentes voláteis presentes na polpa da uva Caimarona foram estudados mediante GC-MS. Estes foram extraídos por três técnicas: evaporação de aroma assistida com solvente (Solvent Assisted Flavour Evaporation SAFE), extração continua líquidolíquido (LL) e destilação por arraste com vapor-extração simultânea com solvente orgânico (DES). Em geral os componentes voláteis predominantes na polpa foram álcoois alifáticos e terpénicos. As notas olfactivas do extracto SAFE foram descritas como floral tenue e verde herbal similares às exibidas pela polpa fresca. Este extrato apresentou como componentes majoritários o linalol 1,2-propanodiol e o salicilato de metilo. Em contraste, o extrato LL apresentou notas de uva passa e vinho moscatel e seus componentes majoritários foram o ácido acético, o salicilato de metilo e o 2,6-dimetil-2(Z),7-octadien-1,6-diol. O extrato DES foi descrito com notas de fresco, floral, cereal e amargo, e foi constituído de um número reduzido de componentes mostrando um efeito negativo da temperatura na extraçáo dos aromas; os componentes majoritários nesta extraçáo foram o 1,2-propanodiol, o linalol e o salicilato de metilo. Adicionalmente, os componentes voláteis majoritários liberados por hidrólises enzimática (Rohapect D5L) dos glicósidos da polpa foram o ácido acético, ácido benzóico e a vanilina. Se deve destacar que ainda que o linalol não esteja entre as agliconas voláteis, notou-se a presença de três dióis biogenéticamente relacionados: o 3,7-dimetil-1,5-octadien-3,7-diol e os isómeros $E$ y $Z$ do 2,6-dimetil-2,7-octadien-1,6-diol.
\end{abstract}

PalaVRaS-CHAVE: Pourouma cecropiifolia; Amazon grape, Uva Caimarona; Cecropiaceae; Compostos voláteis.

\footnotetext{
1 Universidad Nacional de Colombia. E-mail: jcbarriosg@unal.edu.co

2 Universidad Nacional de Colombia. E-mail: dcsinucol@unal.edu.co

${ }^{3}$ Universidad Nacional de Colombia. E-mail: almoralesp@bt.unal.edu.co
} 


\section{INTRODUCCIÓN}

La uva Caimarona (Pourouma cecropiifolia- Martius) es una fruta silvestre de la región amazónica que se distribuye por la cuenca superior del río Amazonas en la parte centrooccidental de la zona compartida por Colombia, Perú y Brasil (Bernal \& Correa, 1991); pertenece a la familia Urticaceae (ex Cecropiaceae) y es un fruto de forma ovoidea-esférica parecida a la uva común pero de mayor tamaño $(2-4 \mathrm{~cm}$ de diámetro) y peso promedio de $15 \mathrm{~g}$. Posee un epicarpio áspero y grueso de color verde en la inmadurez y morado-oscuro al madurar, su pulpa es blanca y mucilaginosa de sabor suave, dulce, agradable, poco ácido y perfumado, en cuyo interior se encuentra la semilla. La pulpa representa el $61 \%$ en peso, el epicarpio 18\% y la semilla el 21\% (Camargo et al., 1991). Debido a las agradables características organolépticas como sabor y textura de la pulpa, ésta se consume en fresco, además se emplea en la elaboración de un licor y las semillas se muelen para preparar una bebida en infusión similar al café.

Aunque los estudios realizados en esta especie son escasos, se ha establecido su valor nutricional en el que se destaca su aporte de calcio, potasio y fósforo, niacina y riboflavina; en un estudio fitoquímico preliminar, mediante pruebas de coloración, se detectaron flavonoides, taninos, triterpenoides y cumarinas (Camargo et al., 1991). Posteriormente, Rivera et al. (2004) estudiaron la actividad de las enzimas polifenol oxidasa y peroxidasa en la pulpa y encontraron que éstas presentan una alta actividad y son las responsables del rápido pardeamiento de la pulpa.

La uva Caimarona es una de las especies catalogadas como frutales económicamente promisorios de la Amazonía que ha sido recomendada para cultivo (Villachica, 1996). A pesar de las agradables características organolépticas de la pulpa y de su potencial económico, dentro de la literatura revisada son escasos los estudios sobre la composición de sus compuestos volátiles libres, Lopes et al., (1999) realizaron una hidrodestilación del fruto sin semilla, originario de la Amazonía brasilera, y mediante el análisis por GC-MS encontraron como componentes volátiles mayoritarios del aceite esencial obtenido, linalol y salicilato de metilo. Recientemente, Pino y Quijano (2008) encontraron estos mismos dos componentes como mayoritarios en un extracto de pulpa colombiana obtenido por destilación extracción simultánea con solvente orgánico (DES). Sin embargo, en estos estudios solo se evalúa un método de extracción y no se ha tenido en cuenta la calidad olfativa de los extractos obtenidos para determinar cuales son los compuestos volátiles activos olfativamente que representan el aroma de esta fruta.

Con respecto a los compuestos enlazados glicosídicamente en la pulpa de la uva Caimarona, no se encontraron estudios publicados. Como continuación de nuestras investigaciones en frutas amazónicas (Fajardo et al., 2005a; Fajardo et al., 2005b) se presenta la composición de los compuestos volátiles libres áctivos olfativamente y de los compuestos volátiles enlazados glicosídicamente en la pulpa de esta fruta.

\section{MATERIALES Y MÉTODOS}

\section{MATERIAL VEGETAL}

Los frutos de uva Caimarona (Pourouma cecropiifolia. Martius) fueron recolectados en el área rural de Florencia (Caquetá, Colombia) en estado de madurez de consumo, epicarpio color morado, $\mathrm{pH} 4.4$ a 4.7 y sólidos solubles 11.8 a 12.0 oBrix (Camargo et al., 1991). Un espécimen fue determinado taxonómicamente en el Herbario Nacional Colombiano del Instituto de Ciencias Naturales de la Universidad Nacional de Colombia donde permanece como testigo bajo el número COL 507603.

\section{AISLAMIENTO DE LOS COMPUESTOS VOLÁTILES LIBRES}

Los compuestos volátiles de la pulpa de esta fruta fueron obtenidos por tres técnicas, extracción continua líquido-líquido (LL), destilación extracción simultanea con solvente orgánico (DES) y evaporación de aroma asistida con solvente (Solvent Assisted Flavour Evaporation SAFE). La primera (LL) ha sido una de las más empleadas para la obtención de extractos de volátiles de frutas, la segunda (DES) permite establecer el efecto de la temperatura sobre la composición de los extractos obtenidos y la última (SAFE) es muy recomendada actualmente porque es una extracción en condiciones suaves que evitan la formación de artefactos y permite obtener los extractos con mayor similitud olfativa con la fruta fresca (Schieberle, 1995).

\section{EXTRACCIÓN LÍQUIDO-LÍQUIDO (LL)}

La extracción se realizó a partir de $500 \mathrm{~g}$ de pulpa de uva Caimarona, la cual se homogenizó a pH natural, la solución resultante se centrifugó a $5000 \mathrm{rpm}$ por 60 minutos $\left(8^{\circ} \mathrm{C}\right)$ y al clarificado se le realizó extracción continua (Parada et al., 2002) con una mezcla de pentano-diclorometano en proporción 1:1 (v/v) por $48 \mathrm{~h}$. La fase orgánica obtenida se secó sobre sulfato de sodio anhidro y se concentró a $45^{\circ} \mathrm{C}$ en un sistema de destilación con una columna Vigreux hasta un volumen de 1 $\mathrm{mL}$. La cuantificación de los compuestos volátiles presentes en el extracto se realizó utilizando 2-heptanol como estándar interno $(213 \mu \mathrm{g} / \mathrm{Kg}$ fruta).

\section{DESTILACIÓN EXTRACCIÓN SIMULTÁNEA CON SOLVENTE ORGÁNICO (DES)}

Para obtener el extracto DES se homogenizaron $500 \mathrm{~g}$ de pulpa a pH natural. Se empleó un extractor tipo LickensNickerson (Flath, Forrey, 1977) y como solvente de extracción una mezcla pentano-éter etílico en proporción 1:1 (v/v); después de $2 \mathrm{~h}$ de extracción la fase orgánica resultante se 
secó sobre sulfato de sodio anhidro y se concentró a $45^{\circ} \mathrm{C}$ en un sistema de destilación con una columna Vigreux hasta un volumen de $1 \mathrm{~mL}$. La cuantificación de los compuestos volátiles presentes en el extracto se realizó con 2-undecanol como estándar interno $(412 \mu \mathrm{g} / \mathrm{Kg}$ fruta).

\section{EVAPORACIÓN DEL AROMA ASISTIDA POR SOLVENTE (SOLVENT ASSISTED FLAVOUR EVAPORATION SAFE)}

La extracción SAFE se realizó con $260 \mathrm{~g}$ de pulpa, la cual se homogenizó a pH natural y se extrajo con $500 \mathrm{~mL}$ de diclorometano durante 2 horas, posteriormente la fase orgánica se pasó por una columna empacada con sulfato de sodio anhidro con el fin de retirar el agua remanente. El extracto obtenido se sometió a la extracción SAFE en un aparato similar al diseñado por Engel et al., (1999). El extracto de volátiles fue concentrado en a $45^{\circ} \mathrm{C}$ en un sistema de destilación con una columna Vigreux hasta un volumen de 1 $\mathrm{mL}$. La cuantificación de los compuestos volátiles presentes en el extracto se realizó con 2-heptanol como estándar interno $(500 \mu \mathrm{g} / \mathrm{Kg}$ fruta $)$.

\section{OBTENCIÓN DEL EXTRACTO DE VOLÁTILES ENLAZADOS GLICOSÍDICAMENTE}

Para la obtención del extracto glicosídico se emplearon 1.5 $\mathrm{Kg}$ de pulpa, se homogenizó con agua y se ajustó el pH a 7.0 con $\mathrm{NaOH}(5 \mathrm{~N})$, la solución resultante se centrifugó a 5000 rpm por 40 minutos a $8{ }^{\circ} \mathrm{C}$. El sobrenadante se sometió a absorción selectiva en una columna de vidrio $(45 \mathrm{x} 450 \mathrm{~mm})$ empacada con Amberlita XAD'-2 (Supelco, Sigma-Aldrich); después de eliminar los azúcares y otro material soluble por lavados sucesivos con agua, los glicósidos se eluyeron con $1 \mathrm{~L}$ de metanol. El solvente se evaporó a presión reducida a $30^{\circ} \mathrm{C}$, los volátiles remanentes se extrajeron con éter etílico y la fase acuosa se liofilizó para obtener $534 \mathrm{mg}$ de glicósidos.

\section{HIDRÓLISIS ENZIMÁTICA}

Una porción del extracto glicosídico $(154 \mathrm{mg})$ se disolvió en $2 \mathrm{~mL}$ de buffer citrato-fosfato a $\mathrm{pH} 5.5$ y se le adicionaron $0.3 \mathrm{~mL}$ de una glicosidasa no selectiva (Rohapect D5L Röhm $\&$ Haas) y fenil $\beta$-D-glucopiranósido como estándar interno $\left(140 \mu \mathrm{g} / \mathrm{Kg}\right.$ fruta). La hidrólisis se llevo a cabo a $37^{\circ} \mathrm{C}$ por 24 horas. Los compuestos volátiles liberados se extrajeron con éter etílico y este extracto se secó con sulfato de sodio anhidro y se concentró a $45^{\circ} \mathrm{C}$ en una columna Vigreux para ser analizado por GC-MS.

\section{CROMATOGRAFÍA DE GASES -ESPECTROMETRÍA DE MASAS} (GC-MS)

Los análisis de los extractos de volátiles obtenidos por LL, DES y el de volátiles liberados por hidrólisis enzimática, se realizaron en un cromatógrafo de gases Hewlett Packard 5890 acoplado a un detector selectivo de masas HP5970 con un sistema de manejo de datos HP5970 MS-Chemstation. Se utilizó una columna capilar de sílica fundida DB-WAX (polietilenglicol, $30 \mathrm{~m} \mathrm{x} 0.25 \mathrm{~mm}$ di, df $0.25 \mu \mathrm{m}$ ). Para el análisis del extracto obtenido por SAFE se empleó un cromatógrafo de gases Shimadzu GC-17A acoplado a un detector selectivo de masas QP5050 con un software CLASS 5000 v 2.2 MSWorkstation y la base de datos espectrales WILEY126. La columna empleada fue DB-FFAP (Polietilenglicol, $30 \mathrm{~m} \mathrm{x}$ $0.32 \mathrm{~mm} \mathrm{di}, \mathrm{df} 0.25 \mu \mathrm{m}$ ). Las condiciones de temperatura en ambos casos fueron: programa de temperatura en el horno $50{ }^{\circ} \mathrm{C}$ durante 4 minutos y ascenso a una velocidad de $4^{\circ} \mathrm{C} /$ minuto hasta $220^{\circ} \mathrm{C}$ donde se mantuvo por 20 minutos. Las temperaturas del inyector y del detector se fijaron en $220^{\circ} \mathrm{C}$. Se utilizó como gas de transporte Helio a $20.7 \mathrm{~cm} / \mathrm{s}$ y 33.9 $\mathrm{cm} / \mathrm{s}$ en las dos columnas, respectivamente. Se trabajó el detector en ionización electrónica con un voltaje de ionización de $70 \mathrm{eV}$, registrando masas entre 40 y $300 \mathrm{u}$. La identificación de los componentes se realizó por comparación de los índices de Kovats y espectros de masas de estándares auténticos ó en su defecto de literatura especializada. La concentración aproximada de los componentes volátiles se realizó de acuerdo con el método del estándar interno sin tener en cuenta los factores de calibración $(F=1.00)$. Los índices de Kovats fueron calculados con respecto a una mezcla de n-alcanos $\mathrm{C}_{10}-\mathrm{C}_{24}$.

\section{CROMATOGRAFÍA DE GASES-OLFATOMETRÍA (GC-0)}

La GC-O se realizó en un cromatógrafo de gases Hewlett Packard 5890 serie II equipado con una columna DB-FFAP (Polietilenglicol, $30 \mathrm{~m} \mathrm{x} 0.32 \mathrm{~mm}$ di, df $0.25 \mu \mathrm{m}$ ) conectada a un divisor de cuarzo (Agilent Technologies PN 5181-3397) que divide el efluente en dos partes iguales mediante dos capilares de sílica fundida desactivada $(50 \mathrm{~cm} \times 0.25 \mathrm{~mm})$ que van hacia el detector FID y hacia el puerto de olfacción. El puerto de olfacción consiste en un tubo de aluminio en forma de codo ( 80 x $5 \mathrm{~mm}$ d.i) montado en la base de un puerto del cromatógrafo y calentado a $200^{\circ} \mathrm{C}$. Durante el análisis GC-O, un panel de expertos entrenados detecta y describe los olores a la salida del puerto de olfacción. Los análisis fueron realizados por tres panelistas entrenados y los resultados se reunieron en un solo cromatograma.

\section{EVALUACION SENSORIAL OLFATIVA}

La pulpa de la uva Caimarona y los extractos derivados de ella fueron evaluados sensorialmente por jueces entrenados. La pulpa fue evaluada en fresco en ausencia del epicarpio y los extractos se evaluaron en cintilla (previa remoción del solvente). 


\section{RESULTADOS Y DISCUSIÓN}

\section{COMPONENTES VOLÁTILES LIBRES}

El aroma de la pulpa de la uva Caimarona fue descrito sensorialmente como floral tenue y herbal verde que recuerda al tomate, a la papa y al lytchee. Aunque el aroma es tenue fue descrito como muy agradable. Los extractos obtenidos por las diferentes técnicas se evaluaron olfativamente, en cintilla; así el extracto DES presentó notas fresca, floral, cereal y fermentado, el extracto líquido-líquido fue descrito sensorialmente con aroma a ciruela, uva pasa y vino moscatel y el extracto SAFE exhibió la mejor calidad olfativa muy similar a la pulpa fresca, con notas florales, verdes y herbales, por tanto este extracto fue analizado por GC-O con el fin de identificar los componentes activos olfativamente.

Los componentes predominantes en todos los extractos fueron alcoholes alifáticos y terpénicos, seguidos por compuestos aromáticos y ácidos alifáticos; sin embargo, la composición de los extractos es cuantitativa y cualitativamente diferente, lo que obedece a las diferencias en las técnicas de extracción (Tabla 1, Figura 1).

El extracto $S A F E$ presentó las características olfativas propias de la pulpa fresca, lo que indica que su composición química es más representativa que la de los extractos obtenidos por las otras metodologías. En este extracto, se destacan el linalol y el propanodiol como componentes volátiles mayoritarios, seguidos por el salicilato de metilo y los isómeros del 2,3butanodiol. Los componentes adicionales se encuentran en concentraciones bajas $(<50 \mu \mathrm{g} / \mathrm{Kg})$. Es importante resaltar que los dioles alifáticos 2,3-butanodiol y propanodiol son producto del metabolismo de azúcares y ácidos alifáticos como tartárico, cítrico y málico, además el primero de éstos es reconocido por ser uno de los componentes que confiere sabor dulce al vino (Catagnia, Avagnina, 2007).

En la evaluación olfativa (GC-O) se destacan zonas del cromatograma con notas florales, herbales y dulces. La nota herbal se debe a los compuestos hexanol y nonanal, el primero recuerda el césped y el segundo es un verde cítrico. El 2,3-butanodiol presentó notas dulces en sus dos conformaciones, el isómero levo presenta nota frutal mientras que el isómero meso tiene una nota que recuerda el aroma del pan y del plátano. Las notas florales son de relevancia sensorial en el extracto y se deben a la presencia del linalol y sus derivados los isómeros $Z$ y $E$ del 2,6dimetil-2,7-octadien-1,6-diol, estos últimos con notas néctar y miel respectivamente y los alcoholes bencílico y feniletílico con notas jazmín y rosa, respectivamente. Si bien en el extracto se encuentran compuestos activos olfativamente ninguno de éstos por si solo posee el aroma de la pulpa fresca (Tabla 2).

El extracto LL presentó como componentes mayoritarios el salicilato de metilo, el (E)-2,6-dimetil-2,7-octadien-1,6diol, el ácido benzoico y el ácido acético. La gran cantidad encontrada de este último $(>150 \mu \mathrm{g} / \mathrm{Kg})$ pone de manifiesto un proceso fermentativo del extracto acuoso de la pulpa durante el proceso de extracción, que puede ser explicado por la alta concentración de azúcares en la pulpa (8.3\%) y las condiciones de la extracción, tiempo $(48 \mathrm{~h})$, temperatura $\left(-20^{\circ} \mathrm{C}\right)$ y $\mathrm{pH}(4.7)$ propicias para la fermentación. En este extracto también se detectaron numerosos ácidos alifáticos y derivados oxigenados del linalol, compuestos que se han encontrado como productos de fermentación de la uva (Vitis vinifera) var. Moscatel (Karagiannis et al., 2000).

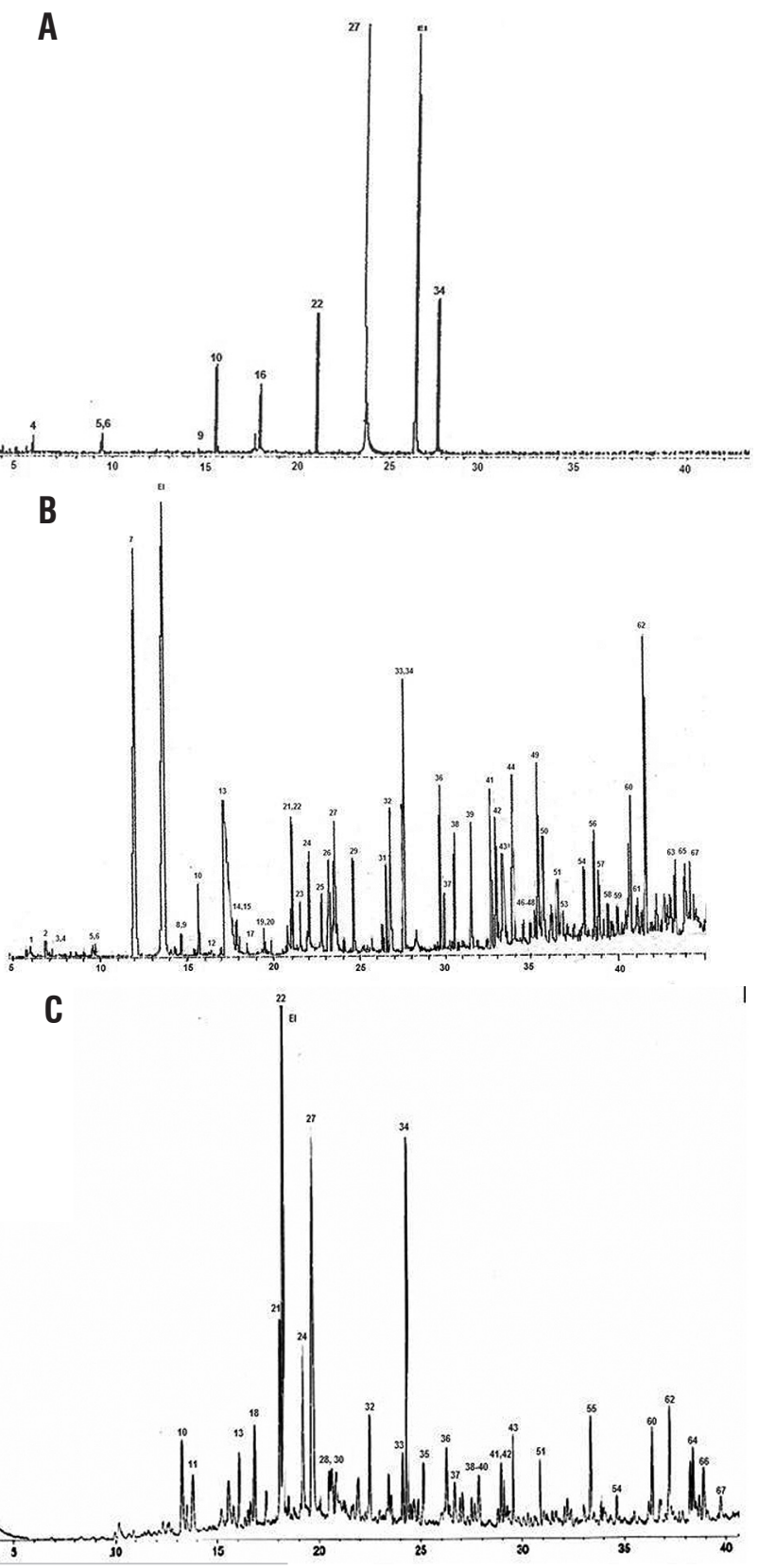

Figura 1 - Cromatogramas de gases de los extractos obtenidos por A. DES B. LL C. SAFE. (A y B, Columna DB-WAX; C. Columna DB-FFAP). 
Tabla 1 - Compuestos volátiles libres detectados en la pulpa de la uva Caimarona por CGAR-EM

\begin{tabular}{|c|c|c|c|c|c|c|}
\hline No & $\mathrm{Ik}_{\text {DB-Wax }}$ & $\mathrm{Ik}_{\text {FFAP }}$ & Compuesto & DES & L-L & SAFE \\
\hline 1 & 1109 & - & 3-pentanol & - & + & - \\
\hline 2 & 1121 & - & 2-pentanol ${ }^{\mathrm{a}}$ & - & + & - \\
\hline 3 & 1134 & - & p-xileno & - & + & - \\
\hline 4 & 1143 & - & butanol & + & + & - \\
\hline 5 & 1207 & - & 3-metil-1-butanol & + & + & - \\
\hline 6 & 1211 & - & (E)-2-hexenal & + & + & - \\
\hline 7 & 1280 & - & 3-hidroxi-2-butanona ${ }^{\text {a }}$ & - & ++ & - \\
\hline 8 & 1341 & - & 2-ciclopenten-1-ona a & - & + & - \\
\hline 9 & 1351 & - & hexanol & + & + & - \\
\hline 10 & 1380 & 1390 & (Z)-3-hexenol & ++ & + & + \\
\hline 11 & - & 1397 & nonanal & - & - & + \\
\hline 12 & 1400 & - & $(E)$-2-hexenol a & - & + & - \\
\hline 13 & 1423 & 1470 & ácido acético & - & ++++ & + \\
\hline 14 & 1443 & - & 1-octen-3-ol a & - & + & - \\
\hline 15 & 1448 & - & $\begin{array}{c}\mathrm{m} / \mathrm{z} 70 \text { (9), } 69(44), 55(21), 43(44), 42(9) \\
41(100), 39(36), 33(13)^{\mathrm{c}}\end{array}$ & - & + & - \\
\hline 16 & 1459 & - & furfural & ++ & - & - \\
\hline 17 & 1460 & - & óxido (E)-furánico de linalol & - & + & - \\
\hline 18 & - & 1488 & $\alpha$-cubebeno ${ }^{\mathrm{a}}$ & - & - & + \\
\hline 19 & 1489 & - & $\begin{array}{c}\mathrm{m} / \mathrm{z} 71(20), 61(7), 45(18), 44(11), 43 \\
(100), 42(9), 41(43), 39(25)^{c}\end{array}$ & - & + & - \\
\hline 20 & 1499 & - & benzaldehido & - & + & - \\
\hline 21 & 1533 & 1563 & 2,3-butanodiol (levo) & - & ++ & ++ \\
\hline 22 & 1539 & 1569 & linalol & +++ & ++ & ++++ \\
\hline 23 & 1552 & - & ácido 2-metilpropanoico a & - & + & - \\
\hline 24 & 1570 & 1604 & 2,3-butanodiol (meso) & - & + & ++ \\
\hline 25 & 1592 & - & 3-metil- $\gamma$-butirolactona ${ }^{\text {a }}$ & - & + & - \\
\hline 26 & 1606 & - & $\gamma$-butirolactona & - & ++ & - \\
\hline 27 & 1618 & 1622 & 1,2-propanodiol & ++++ & ++ & ++++ \\
\hline 28 & - & 1657 & 1,2-etanodiol & - & - & + \\
\hline 29 & 1653 & - & ácido 3-metilbutanoico & - & + & - \\
\hline 30 & - & 1664 & 2-fenilacetaldehido & - & - & + \\
\hline 31 & 1717 & - & carvona & - & + & - \\
\hline 32 & 1728 & 1760 & óxido (Z)-piránico de linalol a & - & ++ & + \\
\hline 33 & 1754 & 1787 & óxido $(E)$ piránico de linalol a & - & ++ & + \\
\hline 34 & 1757 & 1795 & salicilato de metilo & +++ & +++ & +++ \\
\hline 35 & - & 1802 & 2,5-dimetil-2,5-hexanodiol b & - & - & + \\
\hline
\end{tabular}




\begin{tabular}{|c|c|c|c|c|c|c|}
\hline No & $\mathrm{IK}_{\mathrm{DB}-\mathrm{Wax}}$ & $\mathrm{Ik}_{\text {FFAP }}$ & Compuesto & DES & $L-L$ & SAFE \\
\hline 36 & 1833 & 1869 & ácido hexanoico & - & + & + \\
\hline 37 & 1844 & 1885 & guayacol & - & + & + \\
\hline 38 & 1864 & 1897 & alcohol bencílico & - & ++ & + \\
\hline 39 & 1899 & 1931 & alcohol feniletílico & - & ++ & + \\
\hline 40 & - & 1967 & benzotiazol & - & - & + \\
\hline 41 & 1937 & 1973 & 3,7-dimetil-1,5-octadien-3,7-diol a & - & ++ & + \\
\hline 42 & 1946 & 1979 & ácido (Z)-2-hexenoico a & - & ++ & + \\
\hline 43 & 1959 & 1996 & dietilenglicol & - & + & + \\
\hline 44 & 1978 & - & fenol & - & ++ & - \\
\hline 45 & 1998 & - & 2-oxo-pirrolidina a & - & + & - \\
\hline 46 & 2015 & - & $\gamma$-nonalactona ${ }^{\text {a }}$ & - & + & - \\
\hline 47 & 2025 & - & 4-hidroxi-3,6-oxido de linalol a & - & + & - \\
\hline 48 & 2034 & - & ácido octanoico & - & ++ & - \\
\hline 49 & 2048 & - & $\begin{array}{c}\mathrm{m} / \mathrm{z} 115 \text { (15), } 71(47), 69(9), 59(11), 55 \\
(11), 43(100), 41(24), 39(13)^{\mathrm{c}}\end{array}$ & - & ++ & - \\
\hline 50 & - & 2050 & tritertbutilfenol & - & + & + \\
\hline 51 & 2083 & - & $\begin{array}{c}\mathrm{m} / \mathrm{z} 121(11), 69(9), 67(6), 57(7), 55(11) \\
43(100), 41(12), 39(7)^{\mathrm{c}}\end{array}$ & - & + & - \\
\hline 52 & 2095 & - & isobutanoato de 2-fenoxietilo a & - & + & - \\
\hline 53 & 2148 & 2175 & ácido nonanoico & - & + & + \\
\hline 54 & - & 2153 & 2-fenoxietanol a & - & - & + \\
\hline 55 & 2172 & - & 4-vinilguayacol & - & ++ & - \\
\hline 56 & 2183 & - & óxido de $(E)$-epoxilinalol piranoide ${ }^{\mathrm{a}}$ & - & + & - \\
\hline 57 & 2204 & - & palmitato de metilo & - & + & - \\
\hline 58 & 2228 & - & $\begin{array}{l}\mathrm{m} / \mathrm{z} 102 \text { (18), } 71 \text { (16), } 60 \text { (25), } 57 \text { (16), } 55 \\
\text { (21), } 43 \text { (100), } 41 \text { (50), } 39 \text { (18) }\end{array}$ & - & + & - \\
\hline 59 & 2257 & 2284 & 2,6-dimetil-2(Z),7-octadien-1,6-diol & - & - & + \\
\hline 60 & 2276 & - & $\begin{array}{c}\mathrm{m} / \mathrm{z} 60 \text { (78), } 59 \text { (100), } 55 \text { (32), } 43(78), 42 \\
(20), 41(78), 39(34), 31(37)^{\mathrm{c}}\end{array}$ & - & + & - \\
\hline 61 & 2298 & 2322 & 2,6-dimetil-2(E),7-octadien-1,6-diol & - & +++ & + \\
\hline 62 & 2361 & - & 1-hexadecanol & - & + & - \\
\hline 63 & - & 2375 & $\begin{array}{c}\text { m/z } 59 \text { (13), } 58(100), 57(8), 45(16), 43 \\
\text { (77), } 42(10), 41(8), 40(8)^{c}\end{array}$ & - & - & + \\
\hline 64 & 2381 & - & $\begin{array}{c}\mathrm{m} / \mathrm{z} 84(23), 72(41), 71(25), 57(25), 55 \\
(32), 43(100), 41(41), 39(23)^{c}\end{array}$ & - & ++ & - \\
\hline 65 & - & 2397 & $\begin{array}{c}\mathrm{m} / \mathrm{z} 73(7), 59(13), 58(100), 45(13), 44(8) \\
43(87), 42(16), 41(8)^{\mathrm{c}}\end{array}$ & - & - & + \\
\hline 66 & 2395 & 2401 & ácido benzoico & - & +++ & + \\
\hline
\end{tabular}

Concentración (mg/ Kg fruta) $+<50,++50-100,+++100-150,++++>150$;

a. Identificado con base en su índice de Kovats y espectro de masas;

b. Identificado con base en su espectro de masas;

c. Ocho fragmentos de mayor abundancia en el espectro de masas. 
Tabla 2 - Compuestos volátiles activos olfativamente presentes en el extracto SAFE detectados en la pulpa de la uva Caimarona por CGAR-EM

\begin{tabular}{lc}
\hline Compuesto & Nota olfativa \\
\hline (Z)-3-hexenol & verde, césped \\
\hline nonanal & verde, cítrico \\
\hline$\alpha$-cubebeno & herbal, cardamomo \\
\hline 2,3-butanodiol (levo) & dulce, frutal \\
\hline linalol & dulce, cítrico, floral \\
\hline 2,3-butanodiol (meso) & dulce, pan, plátano \\
\hline 2-fenilacetaldehido & azúcar quemado \\
\hline óxido (Z)-piránico de linalol & herbal, cítrico \\
\hline óxido (E) piránico de linalol & frutal \\
\hline salicilato de metilo & medicinal \\
\hline 2,5-dimetil-2,5-hexanodiol & mentolado \\
\hline guayacol & arroz tostado \\
\hline alcohol bencílico & floral, jazmin \\
\hline alcohol feniletilico & rosa \\
\hline benzotiazol & pungente \\
\hline 3,7-dimetil-1,5-octadien-3,7-diol & miel \\
\hline 2,fenoxietanol & floral \\
\hline 2,6-dimetil-2(E),7-octadien-1,6-diol & nectar, floral \\
\hline
\end{tabular}

De acuerdo con las evidencias anteriores puede afirmarse que el menor tiempo empleado en la extracción SAFE (2h) junto con la recuperación de los compuestos volátiles a muy baja temperatura $\left(-60^{\circ} \mathrm{C}\right)$ hacen que la mezcla de compuestos volátiles detectada sea la que representa más fielmente el aroma del material en estudio.

Para observar el efecto de la temperatura en la composición de los componentes volátiles de la pulpa de la uva Caimarona se empleó la extracción DES. Como se mencionó anteriormente, la temperatura empleada tuvo una influencia negativa en las características olfativas del extracto obtenido, fue descrito con notas fresca, floral, cereal y fermentado, muy diferentes a las de la pulpa fresca. Los compuestos mayoritarios en este extracto fueron el propanodiol, el linalol y el salicilato de metilo, estos compuestos también son importantes cuantitativamente en los extractos obtenidos por las otras técnicas. Este resultado indica que estos compuestos son estables a la temperatura de extracción; es importante destacar la presencia de furfural que se produce por la transformación de los azúcares presentes en la pulpa de la uva en las condiciones de $\mathrm{pH}$ y temperatura de la extracción (Quintas et al., 2007).

\section{COMPONENTES VOLÁTILES ENLAZADOS GLICOSÍDICAMENTE}

El extracto de compuestos volátiles obtenido por hidrólisis enzimática (Rohapect D5L) de los glicósidos aislados de pulpa de uva Caimarona presentó 15 compuestos (Tabla 3, Figura 2), siendo los compuestos mayoritarios el ácido acético, el ácido benzoico y la vainillina.

Entre las agliconas liberadas enzimáticamente se encontró el 2,3-butanodiol en conformación meso. Este compuesto se detectó en sus dos conformaciones (meso y levo) en el extracto de volátiles libres SAFE, estos resultados sugieren que este compuesto no sólo es producto del metabolismo de azúcares sino que también puede provenir de un precursor glicosídico y que es posible que ocurra un cambio de conformación en las condiciones trabajadas para la extracción de volátiles libres.

El 2,3-butanodiol se ha encontrado enlazado glicosídicamente con predominancia del isómero meso, en frutas tropicales como la mora de castilla (Rubus glaucus Benth) en la que se detectaron los dos isómeros como agliconas liberadas con una mayor proporción $(300-1000 \mu \mathrm{g} / \mathrm{Kg} \mathrm{de}$ fruta) del isómero meso (Morales et al., 1996) y en la uchuva (Physalis peruviana L.) en la que se detectó únicamente el isómero meso en una concentración superior a $1300 \mu \mathrm{g} / \mathrm{Kg}$ de fruta (Duque et al., 2005).

Aunque uno de los componentes importantes de los extractos de volátiles libres fue el linalol, éste no se detectó entre los compuestos liberados por hidrólisis enzimática de los glicósidos, no obstante, se encontraron dos dioles relacionados biogenéticamente con el linalol, los isómeros $(E)$ y $(Z)$ del 2,6-dimetil-2,7-octadien-1,6-diol. De acuerdo con estudios realizados por Winterhalter et al. (1986), estos dioles están presentes en la papaya fresca pero solo se detectan cuando el proceso de extracción se realiza a pH 7.0 y no al pH natural de ésta (5.6), hecho que demuestra que éstos sufren transformación al $\mathrm{pH}$ natural de la fruta para formar otros compuestos.

Los dos isómeros del 2,6-dimetil-2,7-octadien-1,6-diol han sido encontrados comúnmente como compuestos volátiles libres y enlazados glicosídicamente en frutas donde el linalol es constituyente del aroma libre. Entre éstas, pueden mencionarse la curuba (Passiflora mollissima) (Fröhlich et al., 1989) y la papaya (Carica papaya) (Winterhalter et al., 1986) donde se encuentran como volátiles libres, y el lulo (Solanum vestissimum D.) en el que se hallan como glicósidos en cantidades importantes $(>1000 \mu \mathrm{g} / \mathrm{Kg}$ ) (Suarez, Duque, 1991, Suarez et al., 1993). 


\begin{tabular}{|c|c|c|c|}
\hline No & $\mathrm{IK}_{\text {exp DB-Wax }}$ & compuesto & concentración \\
\hline 1 & 1422 & ácido acético & +++ \\
\hline 2 & 1449 & furfural & + \\
\hline 3 & 1485 & $\begin{array}{c}\text { m/z } 57(8), 46(100), 45(77), 44(20), \\
\quad 43(4), 41(4), 39(3), 31(3)^{\mathrm{a}}\end{array}$ & + \\
\hline 4 & 1504 & benzaldehido & + \\
\hline 5 & 1525 & $\begin{array}{l}\mathrm{m} / \mathrm{z} 45(100), 57(34), 31(21), 73 \\
(11), 74(6), 55(4), 56(4), 42(3)^{\mathrm{a}}\end{array}$ & + \\
\hline 6 & 1556 & 5-metilfurfural & + \\
\hline 7 & 1571 & 2,3-butanodiol (meso) & + \\
\hline 8 & 1615 & ácido butanoico & + \\
\hline 9 & 1657 & ácido 3-metilbutanoico & + \\
\hline 10 & 1685 & 3-metil-2,5-furandiona & + \\
\hline 11 & 1835 & ácido hexanoico & + \\
\hline 12 & 1865 & alcohol bencílico & + \\
\hline 13 & 1938 & 3,7-dimetil-1,5-octadien-3,7-diol & + \\
\hline 14 & 2259 & 2,6-dimetil-2(Z),7-octadien-1,6-diol & + \\
\hline 15 & 2298 & 2,6-dimetil-2(E),7-octadien-1,6-diol & + \\
\hline 16 & 2397 & ácido benzoico & ++ \\
\hline 17 & $>2400$ & vainillina & ++ \\
\hline
\end{tabular}

Concentración (mg/Kg fruta) $+<50,++50-100,+++>100$ a. Ocho fragmentos más abundantes del EM

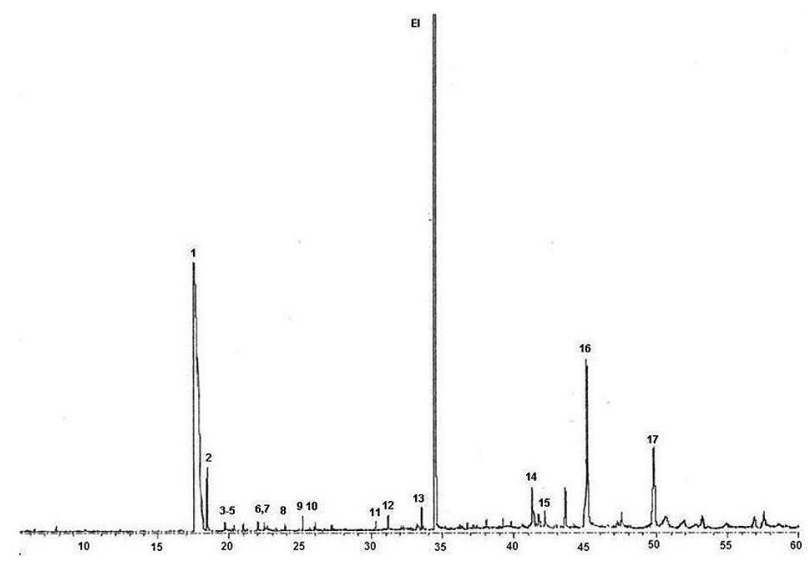

Figura 2 - Cromatograma de gases del extracto de compuestos volátiles liberados por hidrólisis enzimática (Columna DB-Wax)

Es importante resaltar la presencia del 3,7-dimetil-1,5octadien-3,7-diol, éste ha sido encontrado anteriormente en uvas (Vitis vinifera) de la variedad francesa Moscatel, rica en linalol (Gunata et al., 1985) y en Koubo (Cereus peruvianis L.) una fruta que se destaca por su alto contenido de linalol (25 $\mu \mathrm{g} / \mathrm{g}$ de fruta) (Ninio et al., 2003), Aunque este compuesto es encontrado usualmente en frutas con contenidos importantes de linalol, la relación entre estos dioles aún no ha sido establecida.

La presencia de estos dioles monoterpénicos como compuestos volátiles enlazados en la pulpa de la uva Caimarona permite sugerir que sean precursores del linalol, compuesto importante en el aroma de la pulpa de esta fruta.

\section{AGRADECIMIENTOS}

Los autores agradecen el apoyo financiero de Colciencias y de la División de Investigación (DINAIN) de la Universidad Nacional de Colombia y al profesor Alberto Fajardo de la Universidad de la Amazonía por la consecución de las frutas empleadas en este trabajo, adicionalmente a la empresa LUCTA GRANCOLOMBIANA S.A por su colaboración a lo largo del trabajo, a Aleyda Jiménez y Jenny Delgado (Belcorp Colombia) por su colaboración en la evaluación olfatométrica de los extractos.

\section{BIBLIOGRAFIA CITADA}

Bernal, H.Y.; Correa, J.E. 1991. Especies Vegetales Promisorias de los Países Miembros del Convenio Andrés Bello. SECAB (XII), Bogotá, Colombia. 1-20.

Camargo, H.C.; Velásquez, M.A.; Acosta, M. R. 1991. Caracterización de la uva Caimarona (Pourouma cecropiifolia). Colombia Amazónica, 5: 9-26.

Catania, C.; Avagnina, S. 2007. Los estímulos dulces del vino. In: Curso superior de degustación de vinos. Material de curso. EEAMendoza. INTA. Sección 1.5 (http://www. inta.gov.ar/mendoza/invest/Doc_Cursos/5.\%20Los\%20 est $\%$ C3\%ADmulos\%20\%20dulces\%20del\%20vino.pdf) Acceso: 13/12/2008

Duque, C.; Mayorga, H.; Knapp, H.; Winterhalter, P. 2005. Estudios sobre el delicado aroma de uchuva (Physalis peruviana L.) y algunos de sus precursores de tipo glicosídico. En: Duque, C, Morales, AL (Eds). El Aroma Frutal de Colombia. Unibiblos. Universidad Nacional de Colombia, Bogotá, Colombia. p. 48-49.

Engel, W.; Bahr, W.; Schieberle, P. 1999. Solvent Assisted Flavour Evaporation -A new and versatile technique for the careful and direct isolation of aroma compounds from complex food matrices. European Food Research and Technology, 209: 237241.

Fajardo, A.; Morales, A. L.; Duque. C. 2005a. Flavor studies on Amazonian fruits from Colombia. 1. Free and bound volatiles of cocona (Solanum sessiliflorum Dunal)". En: Spanier, AM, Shahidi, F, Parliament, TH, Mussinan, C, Ho, CT, Tratras Contis, E (Eds). Food Flavor and Chemistry. Edited by Special publication-Royal Society of Chemistry. 300, p. 156-163.

Fajardo, A.; Delgado, J. L.; Morales, A. L.; Duque. C. 2005b. Flavor studies on Amazonian fruits from Colombia. 2. Free and bound volatiles of arazá (Eugenia stipitata Mac Vaugh) pulp fruit. In: Spanier, AM, Shahidi, F, Parliament, TH, Mussinan, C, Ho, CT, Tratras Contis, E (Eds). Food Flavor and Chemistry. 
Edited by Special publication-Royal Society of Chemistry. 300, p. 164-174.

Flath, R. A.; Forrey, R.R. 1977. Volatile components of Papaya (Carica papaya L. Solo variety). Journal of Agricultural and Food Chemistry. 22: 103-109.

Fröhlich, O.; Duque, C.; Schereier, P. 1989. Volatile constituents of curuba (Passiflora mollisima) fruit. Journal of Agricultural and Food Chemistry, 37: 421-425.

Gunata, Y.Z.; Bayonove, R.L.; Baumes, R.L.; Cordonnier, R.E. 1985. The aroma of grapes. I. Extracción and determination of free and glycosidically bound fractions of some grape aroma components. Journal of Chromatography, 331: 83-90.

Karagiannis, S.; Economou, A.; Lanaridis, P. 2000. Phenolic and volatile composition of wines made from vitis vinifera $\mathrm{cv}$. muscat lefko grapes from the island of samos. Journal of Agricultural and Food Chemistry, 48: 5369- 5375.

Lopes, D.; Antoniassi, R.; Souza, M. L. M.; Castro, I. M.; Reis, N. S.; Carauta, J. P. P.; Kaplan, M. A. C. 1999. Análise química dos frutos do mapati (Pourouma cecropiifolia Martius- Moraceae). Brazilian Journal of Food Technology, 2: 45-50

Morales, A. L.; Albarracín, D.; Rodriguez, J.; Duque, C.; Riańo, L.; Espitia, J. 1996. Volatile constituents from Andes Berry (Rubus glaucus Benth), Journal of High Resolution Chromatography, 19: 585-587.

Ninio, R.; Lewinsohn, E.; Mizrahi, Y.; Sitrit, Y. 2003. Quality attributes of stored koubo (Cereus peruvianis L. Miller) fruit. Postharvest Biology and Technology, 30: 273-280.

Parada, F.; Bautista, E.; Morales, A. L.; Duque, C. Extractores líquido-líquido para compuestos orgánicos presentes en matrices acuosas. Patente otorgada por Superintendencia de Industria y Comercio (SIC), Res 26812 del 2002, Bogotá- Colombia.
Pino J. A.; Quijano, C. E. 2008. Volatile compounds of Pourouma cecropiifolia Mart. Fruits from Colombia. Journal of Essential Oil Research. 20: 242-244.

Quintas, M.; Guimarães, C.; Baylina, J.; Brandão, T. R. S.; Silva, C. L. M. 2007. Multiresponse modelling of the caramelisation reaction. Innovative Food Science \& Emerging Technologies, 8: 306-315.

Rivera, A. P.; Restrepo, P.; Narváez, C. E. 2004. Polifenoloxidasa y peroxidasa de pulpa de uva Caimarona (Pourouma cecropiifolia Mart.). Revista Colombiana de Química, 33: 57-66.

Schieberle, P. 1995. New developments in methods for analysis of volatile compounds and their precursors. En: Goankar, AG (ed). Characterization of food: emerging methods. Elsevier Science BV. pp. 403-431.

Suárez, M.; C. Duque. 1991. Volatile constituents of lulo (Solanum vestissimun D.) fruit. Journal of Agricultural and Food Chemistry, 39: 1498-1500.

Suárez, M.; Duque, C.; Bicchi, C.; Wintoch, H.; Full G.; Schreier, P. 1993. Volatile constituents from the peelings of lulo (Solanum vestissimun D.) fruit. Flavour and Fragrance Journal, 8: 215220.

Villachica, H. 1996. Frutales y Hortalizas Promisorios de la Amazonía. Tratado de Cooperación Amazónica, Secretaria Pro Tempore, Lima, Perú.

Winterhalter, P.; Katzenberger, D.; Schreier, P. 1986. 6,7-Epoxylinalol and related oxygenated terpenoids from Carica papaya fruit. Phytochemistry, 6: 1347-1350.

Recebido em 27/01/2009

Aceito em 12/05/2009 
